\title{
EFICACIA DE UN PROGRAMA DE EDUCACIÓN SEXUAL EN JÓVENES CON DISCAPACIDAD INTELECTUAL
}

\author{
Mi Ángeles Alcedo Rodríguez* \\ Antonjo-León Aguado Díaz* \\ Benlto Arias Martínez**
}

Departamento de Psicologla. Universidad de Oviedo*

Departamento de Psicologla. Universidad de Valencla*"

\section{RESUMEN}

En este artículo se presenta un programa de intervención psicológica destinado a proporcionar información sobre cuestiones relacionadas con la sexualidad a un grupo de jóvenes con discapacidad intelectual (DI). Dicho programa de educación sexual nace como respuesta a las necesidades planteadas por los profesionales y padres de un grupo de jóvenes con DI pertenecientes a una asociación del Principado de Asturias. En primer lugar, se describen el planteamiento general y los objetivos de dicho programa. En segundo lugar, se detalla el procedimiento de evaluación seguido tanto para la evaluación inicial como para la valoración de resultados y el seguimiento consecuentes. En tercer lugar, se amplía el diseño y la frecuencia del programa y se concretan las técnicas utilizadas en la implementación del mismo. En cuarto lugar, se presentan los resultados alcanzados, cuyos datos muestran diferencias significativas en las respuestas a los items en función de los 
distintos momentos de la evaluación, pretest, posttest y seguimiento. Las diferencias entre el pretest $y$ el posttest indican que la participación en el programa mejora significativamente el nivel de conocimientos sobre cuestiones relacionadas con la sexualidad, mejora que se mantiene a los tres meses de finalizado el programa. Finalmente, el trabajo termina con la discusión de los resultados y una serie de conclusiones acerca de la eficacia y utilidad del programa con el fin de potenciar su implantación.

Palabras clave: DISCAPACIDAD INTELECTUAL, DEFICIENCIA MENTAL, EDUCACIÓN SEXUAL, INTERVENCIÓN, EVALUACIÓN, VALORACIÓN DE RESULTADOS.

\section{SUMMARY}

In this issue a psychological intervention program is presented, aimed at providing information on disability sexuality related issues to a group of youngsters with intellectual disability (ID). Such a sexual education program is bom as a response to the needs raised by professionals and parents of a youngsters group with ID from an association in the Principality of Asturias. Firstly, general approach and objectives of such a program are explained. Secondly, the assessment process used either for the initial assessment or the outcome evaluation as well as the follow up is reported. Thirdly, the program design, frequency and techniques used in its implementation are described. Fourthly, the results obtained are presented. Data show significant differences in items responses according to the assessment different moments, pre-test, post-test and follow up. The differences between pre-test and pos-test point out the significant increasing of acknowledge on sexuality, increasing that is maintained along tree months after the program. Finally, the issue ends with a general discussion about the results and the several conclusions about the program efficacy and utility are commented with the purpose of promoting its implementation

Key word8: INTELLECTUAL DISABILITY; MENTAL DEFCIENCY; SEXUAL EDUCATON; INTERVENTION; ASSESSMENT; OUTCOME EVALUATION. 


\section{INTRODUCCIÓN}

El tema de la educación sexual de las personas con discapacidad intelectual (DI), derecho reconocido por ley desde hace más de tres décadas, y en fechas más recientes de nuevo recogido en la declaración aprobada por la Asamblea General de la Asociación Mundial de Sexologla en el $14^{\prime}$ Congreso Mundial de Sexologfa (1999), viene despertando creciente interés en los profesionales y educadores que atienden a estas personas (Kempton y Kahn, 1991; Brockley, 2002). Además, el hecho de que los frecuentes abusos sexuales que sufre esta población de personas con DI estén estrechamente relacionados con la falta de información y educación sexual acentuó el énfasis y preocupación por el tema (Cambridge y Mellan, 2000; Cooke y Standen, 2002; Valenti-Hein, 2002; Verdugo, Alcedo, Bermejo y Aguado, 2002; Whitehouse y McCabe, 1997). En fechas recientes, la Federación Europea de Personas con Discapacidad (FEPH) y la Confederación Europea de Sindicatos (CES) reclamaron, en su Declaración de Tesalónica de 2003, la necesidad de asegurar la información adecuada en materia de sexualidad y una legislación que solucione los problemas de abuso sexual. Se concreta y demanda que la persona con discapacidad goce del derecho a la autodeterminación en todos los aspectos relativos a su sexualidad, ya sea en cuestiones referentes a preferencia sexual, esterilización o contracepción (López, 2005). Pese a este reconocimiento de derechos y desarrollo de documentos programáticos estamos ante un campo de investigación incipiente, disperso, escaso, carente de datos empíricos y con frecuencia falto de rigor cient/fico (Cambridge y Mellan, 2000; Caspar y Masters, 2001; Garwood y McCabe, 2000; Whitehouse y McCabe, 1997). Ya hace casi dos décadas que Pelechano, del Barrio, Calvo, Diego, González, López, Medrano y Omilla (1986) reconocían la dificultad de los padres para abordar el tema de la sexualidad de sus hijos con DI, asf como la falta de planificación de servicios estatales diversificados, las ideas erróneas, las actitudes rígidas y la falta de información sobre el tema. Por desgracia, el panorama no ha cambiado mucho desde entonces.

La sexualidad humana explica toda una amplia gama de procesos relacionados con la construcción de la identidad personal y sexual, el rol personal, la vinculación afectiva, las relaciones de amistad y 
amor, etc. Sin embargo, la sexualidad de las personas con DI ha sido y sigue siendo un aspecto falto de educación, cuidado y rehabilitación. La reducción de su experiencia sexual, bien a través de la exclusión. el aislamiento o la segregación, tanto en la familia como en los centros e instituciones a los que asisten, ha sido la práctica más habitual. Así pues, la carencia de información y educación sobre cuestiones relacionadas con la sexualidad impide que estas personas desarrollen sus capacidades sexuales y afectivas y aprendan las habilidades necesarias para expresarlas de forma adecuada (Ganwood y McCabe, 2000; Kewman, Warschausky, Engel y Warzak, 1997; McCabe, 1999). Además, y en función de la líteratura disponible, puede afirmarse que una adecuada información y educación en el terreno de la vida sexual y afectiva no sólo aporta conocimientos básicos acerca de estos temas sino que también produce importantes beneficios en varias esferas. A saber: a) favorece una concepción positiva de la sexualidad de forma que se entienda como un elemento básico del desarrollo personal y social, b) fomenta una actitud positiva y de aceptación hacia el propio cuerpo que posibilita el incremento de la autoestima y de la aceptación personal, c) facilita un diálogo abierto que permite la expresión de sentimientos y el intercambio de experiencias $\theta$ ideas acerca de la sexualidad, $d$ auspicia vivencias de relaciones interpersonales equilibradas, saludables y preventivas $y$, e) educa para la correcta toma de decisiones y para asumir la responsabilidad de los propios actos (Bermejo, 1999; Blanchett y Wolfe, 2002; Garwood y McCabe, 2000; Kewman, Warschausky, Engel y Warzak, 1997). Item más, no debemos olvidar que muchas de las conductas sexuales alteradas presentes en estos chicos derivan de la negligencia o falta de atención a las necesidades que aparecen en su esfera sexual (Fierro, 2000).

La solución a todas estas carencias pasa, en primer lugar, por romper con los mitos y prejuicios existentes en tomo al tema de la sexualidad en personas con DI (Faesch, 2002; Milligan y Neufeldt, 2001; Verdugo et al,, 2002). $Y$, en segundo lugar, por la puesta en práctica de programas de educación sexual dirigidos tanto a las propias personas con discapacidad como a padres y profesionales (Cuskelly y Bryde, 2004; Garwood y McCabe, 2000; Löfgren-Martenson, 2004; Lumley y Scotti, 2001; Phillips, Morrison, Davis, 2004). Precisamente, 
el programa de educación sexual que a continuación presentamos nace como forma de dar respuesta a las necesidades planteadas por los profesionales y padres de un grupo de jóvenes con DI pertenecientes a una asociación del Principado de Asturias, y se elabora e implementa en base a los siguientes supuestos, todos ellos ampliamente refrendados en la bibliografía especializada (Cambridge y Mellan, 2000; Caspar y Masters, 2001; Garcla, 2000; López, 2002; May y Simpson, 2003; Marchetti y McCartney, 1990):

- Las personas con DI tienen necesidades emocionales de amar y de ser amados, de mostrar afectos, de establecer relaciones y de expresar su sexualidad.

- El nivel intelectual no es determinante de la capacidad para amar, expresar afecto y cariño, compartir sensaciones corporales, enamorar, seducir, respetar a la pareja, etc.

- La conducta sexual de las personas con DI varía en función de variables tales como edad, grado de deficiencia, conducta adaptativa, habilidades psicosexuales, factores socioambientales, etc.

- El desarrollo sexual $\theta$ interés por la sexualidad no se encuentra especialmente retrasado en comparación con sus pares no discapacitados.

- La mayoría de las personas con DI tienen conciencia de su sexualidad, manifiestan inquietud por el tema y tienen sus experiencias en menor o mayor medida, con menor o mayor grado de satisfacción.

El modelo que subyace al programa se basa en un concepto positivo de salud, en el que prima el derecho de las personas con DI a vivir la sexualidad como una fuente de bienestar y una dimensión más de su calidad de vida (López, 2002). El principal objetivo de este programa se centra, pues, en mejorar el escaso o nulo conocimiento de las cuestiones relacionadas con la sexualidad que poseen la mayoría de los jóvenes con DI. Para ello, interesa, por un lado, presentar datos acerca de la eficacia de dicho programa de educación sexual y, por otro, comprobar si se produce el mantenimiento temporal de esa previsible y esperada mejora de conocimientos sobre cuestiones sexuales. Para alcanzar tales objetivos partimos de las siguientes hipótesis:

- Los jóvenes con DI participantes en el programa de educación sexual presentarán un nivel de información mayor acerca de 
cuestiones básicas relacionadas con la sexualidad. De tal forma que se apreciarán diferencias estadísticamente significativas entre la medida pretratamiento y la primera medida postratamiento, diferencias que serán favorables hacia esta última.

- Asimismo, se prevé un mantenimiento temporal de dicha mejora. En consecuencia, la ganancia conseguida una vez aplicado el programa de tratamiento se mantendrá a lo largo del tiempo, concretamente seis meses después de finalizado el programa.

\section{MÉTODO}

\section{Procedimiento y diseño}

La intervención se llevó a cabo mediante un diseño cuasi-experimental con medidas pre y postratamiento, realizándose un muestreo no probabilístico, o no aleatorio, de carácter incidental. La selección de la muestra se ha realizado en función de una serie de criterios considerados como requisitos básicos para los objetivos de este programa. A saber:

- Todos los sujetos participan en alguno de los programas de formación que se imparten en el centro ocupacional al que acuden y cuentan con suficientes recursos personales, especialmente en lo relativo a comprensión y atención, para responder a las exigencias que implica su trabajo. Se espera que estas capacidades también les permitan seguir y asimilar la exposición y desarrollo de los contenidos a impartir en el programa de educación sexual.

- Han recibido información previa acerca de los objetivos y principales contenidos del programa y decidieron voluntariamente su participación.

- Se ha solicitado consentimiento previo a sus padres y tutores, los cuales también han recibido información acerca de los objetivos y contenidos del programa.

\section{Muestra}

La muestra la componen 28 personas con $\mathrm{Dl}, 13$ chicos $(46,4 \%)$ y 15 chicas $(53,6 \%)$, jóvenes en su mayoria, con edades comprendidas 
entre los 20 y los 40 años. La media de edad es de 27,7 años, con una mediana y una moda de 27 y una desviación típica de 4,5. En cuanto al grado de minusvalía reconocido, el rango medio coincide con el $69,04 \%$, siendo el más alto un $90 \%$ y el más bajo un $37 \%$. En lo relativo a la residencia, según el volumen de población, existe un predominio de personas residentes en núcleos de población de 10.000 6 más habitantes $(78,6 \%)$, es decir, áreas de tipo urbano. Por lo que atañe al tipo de convivencia, todos los sujetos viven con su familia propia, padres y hermanos. Por lo que concieme a su formación profesional, asisten a distintos talleres que se imparten en el centro, concretamente jardinerfa (14,3\%), manipulados $(42,8 \%)$, encuademación $(32,2 \%)$ y programa de garantía social $(10,7 \%)$. Respecto al grado de información que estos jóvenes tienen sobre el tema de la sexualidad, sólo en 8 casos $(28,6 \%)$ admiten conocer algo sobre tales cuestiones, aunque no precisan con claridad qué es lo que saben. Además, $\tan$ sólo en otros 8 casos han hablado con otras personas (padres, hermanos y/o amigos) de temas relacionados con la sexualidad. Asimismo cabe señalar que sólo dos chicas afirman haber realizado algún tipo de revisión ginecológica, y en otros tres casos $(10,7 \%)$ admiten estar tomando anticonceptivos por motivos relacionados con alteraciones en la menstruación. Ninguno de los 28 sujetos ha tenido relaciones sexuales. Por último, y en cuanto a lo que estas personas manifiestan entender por sexualidad, en un $42,9 \%$ no saben precisar o explicar cuál es su vivencia o sentimiento al respecto. Para el $57,1 \%$ restante la sexualidad se entiende como hacer el amor $(17,9 \%)$, tener amigos y relacionarse $(10,7 \%)$, enamorarse $(7,1 \%)$, hablar y aprender sobre sexo $(7,1 \%)$, respetar a los chicos/as $(7,1 \%)$, placer $(3,6 \%)$ y relajación $(3,6 \%)$.

\section{Instrumentos}

Para la recogida de datos relativos al conocimiento e información sobre cuestiones relacionadas con la sexualidad se ha utilizado una entrevista construida ad hoc, que recoge información sobre variables sociodemográficas, clínicas y sobre diferentes aspectos relacionados con la sexualidad. A mayor abundamiento, los principales componentes de esta entrevista son: 
- Datos personales: género, edad, estado civil, lugar de residencia, tipo de convivencia y taller de formación al que acuden en su centro ocupacional.

- Datos clínicos: tipo y descripción de la discapacidad y grado de minusvalía reconocido.

- Tipo y grado de información o formación recibida acerca de temas relacionados con la sexualidad.

- Definición y explicación de diferentes términos y aspectos básicos relacionados con el desarrollo sexual y afectivo: ovario, pene, semen, testiculos, coito, fimosis, erección, eyaculación, masturbación, homosexualidad, menstruación, anticonceptivos, fecundación, embarazo, parto, aborto y enfermedades de transmisión sexual.

Estos 17 términos hacen referencia a cuestiones relacionadas con el desarrollo sexual y las diferentes manifestaciones de la sexualidad, información básica que se adquiere en los primeros años de la infancia y adolescencia. Para facilitar una correcta comprensión de las preguntas se indicaba en primer lugar el término utilizando el vocabulario correcto, por ejemplo, "¿qué es el pene?", y en el caso de que la persona no conociese dicha acepción se preguntaba a través de algunas de las expresiones más frecuentes y cotidianas, incluida la terminología más vulgar con las que habitualmente se hace referencia a dichos conceptos. Para cada uno de los ítems se le pedía a los sujetos una respuesta que definiese el término, en el sentido de qué es, qué significa y a qué hace referencia.

\section{Tratamiento}

Como paso previo a la puesta en marcha del programa se desarrolla una primera fase con el propósito de sensibilizar a los padres y educadores de forma que colaboren y se impliquen en el diseño e implementación del programa. Con este fin se llevan a cabo varias entrevistas y sesiones didácticas con padres y profesores, tanto de forma conjunta como por separado, en las que se plantean como principales objetivos los siguientes: i) consensuar intereses de padres y educadores, ii) determinar la información y contenidos a impartir, iii) establecer prioridades de intervención, iv) fijar criterios educativos a seguir $y, v)$ concretar la duración del programa y la periodicidad de 
las sesiones. Además, tanto a padres como a educadores se les aportó información acerca de cuestiones relevantes sobre la sexualidad en las personas con DI. Dicha información aborda básicamente los siguientes aspectos:

- Ideas generales sobre la sexualidad humana: evolución y desarrollo de la sexualidad; especificidad y peculiaridades que aparecen en las personas con DI.

- Beneficios que la educación sexual aporta al desarrollo personal y emocional de las personas con DI, haciendo especial hincapié en la prevención de abusos sexuales.

- Mitos y creencias falsas sobre los efectos de la educación sexual en personas con DI como, p.e., aumento de su deseo sexual, intento de puesta en práctica de los conocimientos adquiridos, pérdida de control, excitación innecesaria, etc.

En esta fase previa se pretendia transmitir a los padres y educadores la necesidad de que sus hijos y alumnos reciban formación en el área de la sexualidad e involucrarles de alguna manera en el diseño del plan de intervención. Sólo en el momento en el que se consiguió un buen nivel de sensibilización se pasó a la ejecución del programa.

Dado que toda intervención que tenga como objetivo lograr algún tipo de carnbio debe ir precedida de una evaluación previa de la situación y de una valoración posterior que permita comprobar y medir la eficacia de la intervención, la metodologla de trabajo que hemos seguido en la implementación del programa se ha desarrollado a través de una serie de fases. En un primer momento, fase del pretest, se procedió a la aplicación individualizada de la entrevista a los 28 sujetos participantes. Posteriormente se procede a la realización del programa. Se inicia éste con una sesión de presentación de los contenidos y objetivos que se pretenden alcanzar así como de los sujetos participantes. Esta primera sesión, al igual que ocurrirá en las sesiones posteriores, finaliza con una discusión guiada en la que se pide a los jóvenes que planteen preguntas acerca de aquellas cuestiones que más les hayan interesado, que debatan sobre ellas, que expongan dudas y que extraigan las conclusiones más relevantes. Nuestro programa de educación sexual ha tenido un formato grupal y una duración total de 12 sesiones, una por semana, de dos horas de duración cada una de ellas. La aplicación del programa se 
ha realizado en el centro ocupacional al que asisten los sujetos. Se ha utilizado siempre la misma sala, una habitación amplia y bastante aislada de ruidos. El horario de las sesiones es fijo. El entrenamiento está dirigido por un terapeuta, una psicóloga clínica, y una coterapeuta, también licenciada en psicología. Asimismo, para facilitar el contacto y una mejor dinámica del grupo, se cuenta con la presencia de tres educadores que trabajan habitualmente con estas personas y que han decidido voluntariamente participar en el programa como personal de apoyo. La colocación de los miembros del grupo en las sesiones se dispone en forma de semicírculo, situándose el terapeuta y coterapeuta en mitad del grupo. En una pizarra se van anotando las actividades, incldencias y orientaciones más relevantes que acontecen en la sesión. Al inicio de cada sesión se retomen aspectos que puedan haber quedado pendientes y no se avanza en contenidos hasta que dichas cuestiones no están suficientemente aclaradas. La estrategia educativa seguida para la presentación de los contenidos se ha asentado en tres supuestos básicos: i) en la información sobre los distintos temas ha de primar la calidad sobre la cantidad, ii) los contenidos han de presentarse de forma muy estructurada y graduada y, iii) no se irá más allá de lo que estas personas puedan comprender y asimilar. Los procedimientos utilizados para la presentación y explicación de los contenidos han sido variados: descripción oral, escrita y gráfica (textos, fotografías, videos, encuestas, etc.), ensayos de conducta (simulación y dramatización), habilidades de autoconocimiento y reflexión personal, y planteamiento de preguntas y problemas. Todos ellos se han ido combinando a lo largo de las diferentes sesiones. Por último, exponemos a continuación los distintos bloques temáticos y los principales contenidos en los que se ha estructurado la información sobre sexualidad abordada en el programa (cuadro 1).

Una vez finalizado el programa, concretamente una semana después, se procede a valorar la eficacia del mismo aplicando de nuevo la entrevista utilizada en el pretest. Tras este posttest, y pasados seis meses de la finalización del programa, se realiza un seguimiento para controlar sus efectos a largo plazo. De nuevo vuelve a aplicarse la entrevista utilizada en las fases anteriores. 
Cuadro 1. Bloques temáticos y contenidos sobre sexualidad

\section{Somos seres sexuados:}

1.1. Hablemos de sexualidad: qué sé y qué deseo saber

1.2. Distintas manifestaciones de la sexualidad

1.3. Mitos y modelos de belleza

1.4. Desarrollo de un lenguaje básico y correcto

2. Desarrollo sexual:

2.1. Anatomía masculina y femenina: órganos sexuales

2.2. Cambios en la figura corporal durante la adolescencla

2.3. Ovulación y menstruación

2.4. Erección y eyaculación

2.5. Necesidades sexuales

3. Fecundaclón, embarazo y parto:

3.1. Cómo se forma un niño

3.2. Desarrollo del niño durante el embarazo

3.3. Cómo nace

3.4. Distínción entre sexualidad y reproducción

4. Mótodos anticonceptlvos:

4.1. Qué son y para qué sirven

4.2. Cómo acceder a ellos

4.3. Uso correcto de los diferentes métodos

4.4. Riesgos y consecuencias de su utilización

5. HIglene corporal:

5.1. Cuidados básicos del cuerpo

5.2. Higiene e imagen corporal

5.3. Higiene y relaciones interpersonales

6. Enformedades de transmlsión sexual:

6.1. Denominación y vias de transmisión

6.2. Sintomatologla

6.3. Conductas de riesgo

6.4. Medidas preventivas

\section{RESULTADOS}

Interesa ahora proceder al estudio y análisis de las respuestas a los ítems sobre cuestiones sexuales aportadas por los jóvenes con DI participantes en el programa. El principal objetivo es comprobar si 
existen diferencias en las respuestas a los items en función de los distintos momentos de la evaluación, pretest, posttest y seguimiento. Con este fin hemos realizado un análisis unifactorial de la varianza. Para dichos análisis adoptamos el nivel de significación a priori de alfa $=, 05$. Tras comprobar el cumplimiento de los supuestos paramétricos de normalidad (mediante la prueba $z$ de Kolmogorov-Smimov) y de homogeneidad de varianzas (mediante la prueba de Levene) hemos procedido al análisis. Como se pone de manifiesto en la tabla 1, se han encontrado diferencias altamente significativas en todos los ítems.

Tabla 1. Diferencias on las respuestas a los ftems en función de los distintos momentos de la evaluación: pretest, posttest y seguimiento (ANOVA unifactorial)

\begin{tabular}{lcc}
\hline \multicolumn{1}{c}{ Items: } & $F$ & Sig. \\
\hline 1. Ovario & 7,435 & .001 \\
2. Pene & 5,684 & .005 \\
3. Sémen & 11,809 & .000 \\
4. Testículos & 5,442 & .006 \\
5. Coito & 14,570 & .000 \\
6. Fimosis & 4,267 & .017 \\
7. Erección & 23,652 & .000 \\
8. Eyaculación & 10,008 & .000 \\
9. Masturbación & 9,941 & .000 \\
10. Homosexualidad & 3,977 & .023 \\
11. Menstruación & 25,071 & .000 \\
12. Antoconceptivos & 15,848 & .000 \\
13. Fecundación & 4,704 & .012 \\
14. Embarazo & 8,150 & .001 \\
15. Parto & 13,469 & .000 \\
16. Aborto & 3,131 & .049 \\
17. Enfermedades sexuales & 18,708 & .000 \\
\hline
\end{tabular}

Tras este análisis global y al objeto de comprobar entre qué momentos concretos se observan tales diferencias asi como la cuantia de las mismas, hemos realizado la prueba post-hoc de comparaciones múltiples de Scheffé, cuyos resultados presentamos en la tabla 2. 
Tabla 2. Prueba post-hoc de comparaciones múltiples de Scheffé

\begin{tabular}{lccccccc}
\hline \multicolumn{1}{c}{ Items: } & \multicolumn{2}{c}{ Pretest-Postlest } & Pretest-Seguimi. & \multicolumn{2}{c}{ Posttest-Seguiml. } \\
\hline & Difer. M & Sig. & Difer. M & Sig. & Difer. M & Sig. \\
1. Ovario & -.4286 & $.004^{*}$ & -.3929 & $.008^{*}$ & .0357 & .959 \\
2. Pene & -.2143 & .247 & -.4286 & $.005^{*}$ & -.2143 & .247 \\
3. Sémen & -.5357 & $.000^{*}$ & -.4643 & $.001^{*}$ & .0714 & .837 \\
4. Testiculos & -.3929 & $.011^{*}$ & -.3214 & $.046^{*}$ & .0714 & .854 \\
5. Coito & -.5357 & $.000^{*}$ & -.5000 & $.000^{*}$ & .0357 & .950 \\
6. Fimosis & -.1786 &. .275 & -.3214 & $.018^{*}$ & -.1429 & .436 \\
7. Erección & -.7143 & $.000^{*}$ & -.5357 & $.000^{*}$ & .1786 & .261 \\
8. Eyaculación & -.4643 & $.000^{*}$ & -.3571 & $.006^{*}$ & .1071 & .617 \\
9. Mastumación & -.5357 & $.000^{*}$ & -.2500 & .122 & .2857 & .065 \\
10. Homosexualidad & -.3571 & $.024^{*}$ & -.2143 & .249 & .1429 & .536 \\
11. Menstruación & -.7143 & $.000^{*}$ & -.5357 & $.000^{*}$ & .1786 & .241 \\
12. Anticonceptivos & -.6429 & $.000^{*}$ & -.3214 & $.023^{*}$ & .3214 & $.023^{*}$ \\
13. Fecundación & -.3214 & $.024^{*}$ & -.2857 & $.050^{*}$ & .0357 & .953 \\
14. Embarazo & -.4643 & $.002^{*}$ & -.3929 & $.009^{*}$ & .0714 & .847 \\
15. Parto & -.5714 & $.000^{*}$ & -.4643 & $.001^{*}$ & .1071 & .659 \\
16. Aborto & -.1429 & .543 & -.3214 & $.050^{*}$ & -.1786 & .386 \\
17. Enfermedades sexuales & -.6071 & $.000^{*}$ & -.5714 & $.000^{*}$ & .0357 & .950 \\
\hline
\end{tabular}

Si atendemos al primer grupo de comparación, pretest y posttest, observamos que en 14 de los 17 items las diferencias resultan estadísticamente significativas a favor de la segunda medida, es decir, del posttest. Los tres ítems que no presentan cambios significativos entre ambas medidas son el 2 (pene), el 6 (fimosis) y el 16 (aborto). Por tanto, en general, y según indica la prueba post-hoc, los sujetos participantes en el programa puntúan más alto en el posttest que en el pretest en prácticamente todas las preguntas sobre sexualidad recogidas en la entrevista. Este aumento significativo de las medias a favor del posttest apoya nuestra primera hipótesis de que la participación en el programa de educación sexual aportará un mayor nivel de información acerca de cuestiones básicas relacionadas con la sexualidad.

Por lo que concieme al segundo grupo de comparación, pretest y seguimiento, observamos que en general las diferencias se mantienen, 
ya que salvo en dos ítems las diferencias resultan estadísticamente significativas a favor del seguimiento. En la tabla 2 observamos que los sujetos de nuestra muestra puntúan más alto en la segunda medida postratamiento, realizada 6 meses después de la finalización del programa, en 15 de los 17 ítems recogidos en la entrevista. En dos de estos ítems, concretamente en el 9 (masturbación) y en el 10 (homosexualidad), aunque la media de puntuaciones sigue siendo más elevada en el seguimiento que en el pretest, las diferencias no alcanzan significación estadística. También es preciso destacar que los tres ftems que no alcanzaban significación estadística al comparar el pretest y el posttest, hablamos del ítem 2 (pene), del 6 (fimosis) y del 16 (aborto), sí la encuentran en este segundo nivel de comparación. Este aumento significativo de medias a favor del seguimiento apoya nuestra segunda hipótesis de trabajo que planteaba que la ganancia conseguida una vez aplicado el programa de tratamiento se mantendría a lo largo del tiempo, concretamente seis meses después de finalizado el programa. Esta hipótesis también se encuentra avalada por los resultados encontrados en el siguiente grupo de comparación, posttest y seguimiento.

Respecto a este tercer nivel de comparación entre las medidas del posttest y del seguimiento, observamos que, salvo en uno de los ítems, en concreto el número 12 (anticonceptivos), la prueba post-hoc no encuentra diferencias significativas entre ambas medidas. En este análisis comparativo encontramos que tres ítems presentan en el seguimiento puntuaciones más elevadas que en el posttest. Ahora bien, cuando analizamos estas diferencias vemos que en dos de estos ítems, el 2 (pene) y el 6 (fimosis), no alcanzan significación estadística. Representan variaciones normales que se pueden estimar por el azar. Sin embargo, para el ftem 12 (anticonceptivos) las diferencias a favor del seguimiento nos indican que sobre esta cuestión la información que nuestros chicos con DI han podido obtener por otras vías es la que establece dichas diferencias. Salvo esta excepción, los resultados alcanzados en el resto de los ítems se mantienen seis meses después de finalizado el programa. Suponen, por tanto, un nuevo apoyo a la segunda hipótesis que postulaba el mantenimiento temporal de la mejora en el nivel de información adquirido por nuestros sujetos sobre cuestiones relativas a sexualidad. 
Así pues, el análisis de comparaciones múltiples de Scheffé confirma el patrón esperado en la medida en que entre seguimiento y posttest no encuentra diferencias para la mayoría de los ítems pero sí entre ambos y el pretest. Estos resultados confirman la existencia de diferencias en el nivel de información que adquieren los sujetos tras participar en el programa, en el sentido de que antes de su inicio el nivel de conocimientos del que partían era muy bajo, apreciándose posteriormente, una vez implementado el programa y también durante el perlodo de seguimiento, un aumento muy significativo en el conocimiento de cuestiones vanadas relacionadas con el desarrollo y funcionamiento afectivo-sexual, temas todos ellos abordados y desarrollados en el programa.

\section{DISCUSIÓN}

Pese a que está fuera de toda duda que un ambiente sexual normalizado y una adecuada información sobre aspectos relativos al desarrollo y funcionamiento sexual aporta efectos muy positivos al desarrollo personal de las personas con $\mathrm{DI}$, la planificación e intervención en esta área es muy limitada. La escasez de programas y de material educativo elaborado en base a las necesidades específicas de estas personas entorpece la toma de decisiones acerca de qué contenidos y qué tipo de estrategias educativas han de ser utilizadas (Blanchett y Wolfe, 2002). Este fue precisamente el primer escollo al que tuvimos que enfrentamos. Tras una exhaustiva revisión de los programas recogidos en la bibliografía especializada nos decantamos por un programa comprensivo, que abarca diversos contenidos y áreas temáticas (cuadro 1), $\theta$ implementado a través de estrategias educativas variadas (textos, fotografías, vídeos, ensayos de conducta, discusiones guiadas, etc.), ya que éstas parecen ser las que han demostrado mejor eficacia (Blanchett, 2000; Garwood y McCabe, 2000; Blanchett y Wolfe, 2002). Otro inconveniente añadido fue el de encontrar un método de evaluación que nos permitiera obtener una información fiable y válida. Partiendo de la dificultad que conlleva la recogida de información procedente de personas con DI que presentan limitaciones cognitivas y de lenguaje importantes, y ante la carencia de procedimientos de evaluación estandarizados y 
adaptados a nuestros objetivos, hemos optado por elaborar una entrevista que recoja los principales contenidos abordados en el programa. Las recomendaciones de algunos autores (McCabe, Cummins y Deeks, 1999; Blanchett y Wolfe, 2002) abogan por esta técnica de entrevista frente a otro tipo de técnicas, pues consideran que aunque no está exenta de problemas sí evita algunos sesgos muy frecuentes en las respuestas dadas a los autoinformes con alternativa de respuesta dicotómica o escalar por las personas con DI, como es el caso de la aquiescencia o la dificultad para saber con exactitud si el sujeto ha entendido el contenido del ítem al que ha dado respuesta.

Tras esta toma de decisiones se procedió a la ejecución del programa. Los resultados obtenidos han puesto de manifiesto que la conducta sexual de estas personas puede ser educada, al igual que lo es cualquier otro tipo de conducta. El desconocimiento tan profundo que nuestros sujetos presentaban acerca de las cuestiones más básicas relacionadas con la sexualidad pudo ser reemplazado, tras un entrenamiento diseñado y elaborado ad hoc en función de sus intereses y capacidades de aprendizaje, por un nivel de información y conocimiento bastante aceptable. Asi, si procedemos a un análisis detallado de las diferencias en las respuestas dadas a los 17 items recogidos en la entrevista, comprobamos que en el pretest sólo tres ítems, concretamente el 2 (pene), el 4 (testiculos) y el 9 (masturbación), alcanzan la media de puntuaciones más altas, $M=1,32$ (el rango de puntuaciones va de 1 a 2), lo que significa que son conceptos conocidos por sólo 9 de los 28 sujetos que asisten al programa. El resto de las puntuaciones alcanzan valores aún más bajos. De hecho la mayorla de los ítems presentan medias en torno a 1.17, lo que refleja el conocimiento de los mismos por solo 5 sujetos. Es más, cinco de los ítems recogidos en la entrevista, el 5 (coito), el 6 (fimosis), el 7 (erección), el 8 (eyaculación) y el 13 (fecundación), son cuestiones a las que ninguno de nuestros sujetos responde de forma correcta. $\mathrm{O}$ bien son totalmente desconocidos o la información que poseen al respecto es errónea. Por el contrario, en el posttest ya nos encontramos con 12 de los 17 ítems con medias por encima de 1.53, lo que supone que al menos más de la mitad de los sujetos ya dispone de información adecuada sobre dichos temas. 
También interesa el análisis cualitativo de las respuestas dadas en el pretest a algunos de las cuestiones. A modo de ejemplo, baste destacar que hacia la homosexualidad y la masturbación aparecen respuestas cargadas de sentimientos negativos, mitos, concepciones erróneas y miedos. Prima la idea de que la masturbación es algo nocivo y que es una práctica que sólo realizan los hombres. Similares connotaciones se asocian a la homosexualidad, orientación sexual que consideran inadecuada y "mala". El conocimiento acerca de las enfermedades de transmisión sexual y de la contraconcepción era también mínimo. Las creencias erróneas acerca del proceso de transmisión de enfermedades como el SIDA eran las más frecuentes. La opinión más compartida relacionaba beso y saliva como las principales vias de contagio. Acerca de los métodos anticonceptivos la información era asimismo muy limitada y sólo conocían el preservativo, aunque muy pocos lo habian observado de cerca. La función de la pildora se asociaba casi de forma exclusiva a la regulación de los desarreglos menstruales. En relación con los órganos sexuales, sólo identificaban de forma correcta la parte corporal correspondiente pero no conocían o bien describlan erróneamente sus funciones. Sivva, como ejemplo, el hecho de que acerca de la vagina y del pene la mayorla de los sujetos describen su utilidad para orinar y el género al que pertenecen, pero no reconocen explicitamente su función sexual. En lo que concierne al proceso de fecundación, embarazo y parto, sus conocimientos se limitaban a saber que "los bebés crecían dentro del vientre materno y también nacían por el vientren. La vagina, p.e., no solla asociarse al parto. Interesa destacar que todas estas características de respuesta presentes en nuestros sujetos también aparecen recogidas en las principales revisiones sobre programas de educación sexual en personas con DI (Blanchett, 2000; Blanchett y Wolfe, 2002; Cambridge y Mellan, 2000; Caspar y Masters, 2001; Galea, Butler, lacono y Leighton, 2004; Garwood y McCabe, 2000).

Ya en el posttest, así como en el seguimiento, la mayoría de las respuestas a estas cuestiones se habian modificado y nuestros sujetos informaban sobre ellas con más certeza, precisión y profundidad. Es más, quisiéramos anotar que estos cambios en el nivel de información adquirido tienen su reflejo en las opiniones que en el posttest $y$ en el seguimiento expresan ante la pregunta ¿qué es para ti la 
sexualidad? Ya comentamos anteriormente que en el pretest un $42,9 \%$ de estos jóvenes no fueron capaces de explicar cuál era su vivencia o sentimiento al respecto. Las respuestas más frecuentes eran del tipo de "no sabemos cómo decirlo", "eso nunca lo he pensado y no sé qué decir", "de eso mejor ni hablar", etc. En cambio, en el posttest, sólo un $10,7 \%$ de los sujetos deja sin respuesta esta cuestión. En el seguimiento, el porcentaje de sujetos que no responde se reduce a un $3,6 \%$. Parece, pues, que la participación en el programa, la oportunidad de conocer, abordar y discutir estos temas ha posibilitado el que nuestros sujetos con DI puedan expresar lo que sienten sobre su vida sexual y afectiva. O quizás, como apuntan Blanchett y Wolfe (2002), lo que también pudo haber ocurrido es que han perdido el miedo a hablar acerca de estos temas. De hecho, queremos hacer hincapié en la facilidad y naturalidad con que desde un principio el grupo pregunta y se interesa abiertamente por las cuestiones más variadas relacionadas con la vida sexual y afectiva, siempre en un clima de gran confianza y respeto. A medida que avanzaba el programa manifestaban y expresaban sin tapujos sus sentimientos, intercarnbiaban experiencias $\theta$ ideas, se asentaba el respeto a opiniones diferentes, se promovían comportamientos no discriminatorios entre sexos y reflexionaban acerca de las consecuencias de los actos y decisiones personales. Es más, el interés por esta temática se hizo claramente patente a través de las preguntas y consultas que planteaban nuestros sujetos a sus monitores y padres. Una vez finalizado el programa demandaban con frecuencia la necesidad de contar con un espacio en el que poder abordar y debatir con cierta regularidad estas cuestiones. Por tanto, junto al criterio experimental de eficacia que nos aporta la significación empírica del cambio observado como resultado de haber aplicado el programa, también contamos con el criterio clínico o terapéutico, manifestado no solo por los sujetos, sino también, y de forma especial, por los monitores del centro ocupacional al que asisten. Todos reconocen la importancia subjetiva y social de los cambios operados.

\section{CONCLUSIONES Y SUGERENCIAS}

Pese a que ya hace tiempo que está fuera de toda duda que la sexualidad desempeña un papel muy importante en la conformación 
psicologica del ser humano (Pelechano et al., 1986), aún es necesario seguir haciendo hincapié en que la persona con Dl es un sujeto de derechos y deberes y, como tal, se le debe proporcionar todas aquellas oportunidades que faciliten y posibiliten su integración y nomalización. Entre estas oportunidades se encuentra la educación sexual. Ya hace muchas décadas que se viene trabajando en pro de su integración escolar, laboral, social, etc., pero nos estamos olvidando de una dimensión que resulta vital para su desarrollo personal, su sexualidad. Estas personas, para poder adaptarse a las situaciones relacionadas con la experiencia y la actividad sexual, necesitan recibir educación y formación al respecto. Sin embargo, los programas de educación sexual desarrollados e implementados de forma regular son muy escasos, pese a saberse que el conocimiento y la información que al respecto poseen es muy limitada.

El trabajo que hemos presentado, en la línea de otros similares (Blanchett y Wolfe, 2002; Garwood y McCabe, 2000; McCabe, 1999; Wiley y Terlosky, 2000), demuestra que la sexualidad de las personas con DI es educable, es decir, susceptible de abordaje pedagógico. Las personas participantes en nuestro estudio presentaban un nivel de información muy pobre sobre cuestiones básicas relacionadas con la sexualidad. Las diferencias entre el pretest y el posttest indican que la participación en el programa mejora significativamente el nivel de sus conocimientos, mejora que se mantiene a los tres meses de finalizado el programa. Los datos recogidos muestran que estos jóvenes con DI llegan a adquirir y comprender las pautas básicas que guían el desarrollo sexual, los procesos de reproducción, los métodos anticonceptivos, los hábitos de higiene y cuidado corporal y genital, y las enfermedades de transmisión sexual. Sin duda que los resultados presentados adolecen de problemas y limitaciones, y que aún son muchas las cuestiones pendientes de abordaje, pero creemos que suponen una aportación a un campo muy limitado de publicaciones sobre procedimientos de intervención y valoración de la eficacia de este tipo de programas. Probablemente nuestros resultados puedan mejorarse si se aumenta el número de sesiones de aprendizaje. Puede resultar una estrategia adecuada a este fin la programación de sesiones alternativas en el tiempo para afianzar los conocimientos 
adquiridos y apoyar en la resolución de dudas y.problemas que se vayan planteando, ya que parece que el conocimiento sexual de estas personas mejora en proporción directa a la intensidad del programa (Caspar y Masters, 2001). Asimismo, también interesa desarrollar programas específicos que aborden temas que resultan de vital importancia para la integración personal y social de estos chicos y que no suelen ser abordados con profundidad suficiente en los programas al uso (Whitehouse y McCabe, 1997). Asl, las habilidades sociosexuales, las enfermedades de transmisión sexual y el uso de métodos anticonceptivos son áreas que precisan un abordaje intenso.

Y ya para finalizar, sólo nos queda abogar por el diseño y ejecución de programas de educación sexual, dirigidos tanto a los propios sujetos con DI como a los padres y profesionales que les atienden, para asi conseguir incrementar ese objetivo central en torno al cual gira toda intervención en el ámbito de la discapacidad intelectual, la mejora de la calidad de vida de los sujetos afectados. Esperemos que todo ello ayude a desarrollar en estos jóvenes una concepción positiva de la sexualidad y de sus diversas manifestaciones, alejada de los múltiples prejuicios y estereotipos a que viene estando sometida.

\section{REFERENCIAS BIBLIOGRÁFICAS}

Asociación Mundial de Sexología (1999). Declaración de los Derechos Sexuales. $14^{9}$ Congreso Mundial de Sexología, Asamblea General de la Asociación Mundial de Sexología. Hong Kong, República Popular China. (Manuscrito xerocopiado).

Bermejo, B.G. (1999). El desarrollo de habilidades sociales como forma de prevenir el abuso sexual en personas con discapacidad. Siglo Cero, 30 (6), 27-31.

Blanchett, W.J. (2000). Sexual risk behaviors of young adults with LD and the need for HIV/AIDS education. Remedial and Special Education, 21, 336-345.

Blanchett, W.J. y Wolfe, P.S. (2002). A review of sexuality education curricula: Meeting the sexuality education needs of individuals with moderate and severe. Research and Practice for Persons with Severe Disabilities, 27 (1), 43-57. 
Brockley, J. A. (2002). Rearing the child who never grew: Parents, professionals, and children with intellectual disabilities, 1910-1965. Dissertation Abstracts Intemational Section A: Humanities and Social Sciences, 62 (10-A), 3535.

Cambridge, P. y Mellan, B. (2000). Reconstructing the sexuality of men with learning disabilities: empirical evidence and theoretical interpretations of need. Disability and Society, 15 (2), 293-311.

Caspar, L.A. y Masters, L. (2001). Sexuality education for adults with developmental disabilities. Education and training in mental retardation and developmental disabilities, 36 (2), 172-177.

Cooke, P. y Standen, P.J. (2002). Abuse and disabled children: hidden needs...?. Child Abuse Review, 11 (1), 1-18.

Cuskelly, M. y Bryde, R. (2004). Attitudes towards the sexuality of adults with an intellectual disability: Parents, support staff, and a community sample. Joumal of Intellectual and Developmental Disability, 29 (3), 255-264.

Faesch, C. (2002). Sexualite et Handicap: Briser le Tabou. ETRE, 58, 6-9.

Fierro, A. (2000): El sexo de los ángeles. En J.R. Amor (Ed): Sexualidad y personas con discapacidad psiquica, (ps.51-67). Madrid: FEAPS.

Galea, B., Butler, J., lacono, T. y Leighton, D. (2004). The assessment of sexual knowledge in people with intellectual disability. Joumal of Intellectual and Developmental Disability, 29 (4), 350-365.

García, J.L. (2000). Educación sexual y afectiva en personas con minusvalía psíquica. Cádiz: Asociación Síndrome de Down Cádiz y Bahía.

Garwood, M. y McCabe, M. (2000). Impact of sex education programs on sexual knowledge and feelings of men with a mild intellectual disability. Education and Training in Mental Retardation and Developmental Disabilities, 35 (3), 269-283.

Kempton, W. y Kahn, E. (1991). Sexuality and people with intellectual disabilities: A historical perspective. Sexuality and Disability, 9 (2), 93-111.

Kewman, D., Warschausky, S.; Engel, L. y Warzak, W. (1997). Sexual development of children and adolescents. En M. Sipski y A. Craig (Ed): Sexual function in people with disability and chronic illness: 
A health professional's guide, (ps. 335-378). Gaithersburg, MD, USA: Aspen Publishers.

Löfgren-Martenson, L. (2004). May I?" About sexuality and love in the new generation with intellectual disabilities. Sexuality and Disability, 22 (3), 197-207.

López, F. (2002). Sexo y afecto en personas con discapacidad. Madrid: Biblioteca Nueva.

López, M. (2005). Personas, derechos y servicios. En M. López y R.

Carbonell (Coors): La integración educativa y social, (ps.31-65). Barcelona: Ariel.

Lumley, V.A. y Scotti, J.R. (2001). Supporting the sexuality of adults with mental retardation. Current status and future directions. Joumal of Positive Behavior Interventions, 3 (2), 109-119.

May y Simpson, (2003). The parent trap: marriage, parenthood and adulthood for people with intellectual disabilities. Critical Social Policy, 23 (1), 25-43.

Marchetti, A.G. y McCartney, J.R. (1990). Abuse of persons with mental retardation: Characteristics of the abused, the abusers, and the informers. Mental Retardation, 28, 367-371.

McCabe, M. (1999). Sexual knowledge, experience and feelings among people with disability. Sexuality and Disability, 17 (2), 157-170.

McCabe, M., Cummins, R.A. y Deeks, A. (1999). Construction and psychometric properties of sexuality scales: Sex Knowledge, experience, and needs for people with intellectual disabilities (Sex Ken-PD), and the general population (Sex Ken-GP). Research in Developmental Disabilities, 20, 241-254.

Milligan, M.S. y Neufeldt, A.H. (2001). The myth of asexuality: a survey of social and empirical. Sexuality and Disability, 19 (2), 91-110.

Phillips, Morrison, Davis, (2004). General practitioners' educational needs in intellectual disability health. Joumal of Intellectual Disability Research, 48 (2), 142-149.

Pelechano, V., Del Barrio, J.A., Calvo, G. Diego, R. González, M., López Dóriga, M.J., Medrano, P. y Omilla, I. (1986): Actitudes y cambio de actitudes sobre la sexualidad en padres de deficientes mentales. Análisis y Modificación de Conducta, 12 (31-32), 263-280.

Valenti,-Hein, D. (2002). Use of visual tools to report sexual abuse for adults with mental retardation. Mental Retardation, 40 (4), 297-303. 
Verdugo, M.A., Alcedo, M.A., Bermejo, B. y Aguado, A.L. (2002). Abuso sexual en personas con discapacidad intelectual. Psicothema, 14 (suplemento), 124-129.

Whitehouse, M.A. y McCabe, M.P. (1997). Sex education programs for people with intellectual disability: how effective are they? Education and Training in Mental Retardation and Developmental Disabilities, 32, 229-240.

Wiley, D.C. y Terlosky, B. (2000). Evaluating sexuality education curriculums. Association for Supervision and Curriculum Development, 58, 79-82. 